\title{
Microcirculation: No Excuses Left for not Assessing!
}

\author{
Olcay Dilken and Can Ince* \\ Department of Intensive Care, Erasmus MC, The Netherlands \\ *Corresponding author: Can Ince, Department of Intensive Care, Erasmus MC, The Netherlands
}

\begin{tabular}{ll}
\hline ARTICLE INFO & ABSTRACT \\
\cline { 1 - 1 } $\begin{array}{l}\text { Received: } \\
\text { Published: August 02, } 2019\end{array}$ & $\begin{array}{l}\text { The microcirculation is vital in providing essential components throughout the } \\
\text { organism. It is also delicate and deteriorates early in shock patients. Therapies aimed } \\
\text { at improving macrohemodynamic values are expected to improve microcirculation also. }\end{array}$ \\
$\begin{array}{ll}\text { However, there is an increased evidence that loss of hemodynamic coherence may occur } \\
\text { Citation: Olcay Dilken, Can Ince. Micro- } \\
\text { circulation: No Excuses Left for not As- } \\
\text { sessing!. Biomed J Sci \& Tech Res 20(2)- }\end{array}$ & $\begin{array}{l}\text { in some shock patients. This makes monitoring of the microcirculation an important step } \\
\text { in managing shock patients. The newly introduced software substantially decreases the } \\
\text { workload imposed on the operator }\end{array}$
\end{tabular}
2019. BJSTR. MS.ID.003439.

\section{Introduction}

Microcirculation is crucial in delivering oxygen, nutrients, regulating immune functions and removal of the waste products [1]. Vessels smaller than $20 \mu \mathrm{m}$ in diameter constitutes this network. Normally, microcirculation holds around $10 \%$ of the total blood volume. As it is involved in vital functions, its monitoring in critical illness is deemed essential [2]. Shock is common amongst Intensive Care Unit (ICU) Patients. Regardless of the shock type, fluid administration and inotropic and/or vasopressor drugs remain the backbone of the therapy. This modality aims for the normalization of macrohemodynamic parameters, such as blood pressure and cardiac output. It is anticipated that an improvement in these parameters will result in an improvement in the microcirculation also. However, it was shown that it is not the case in shock patients. This mismatch between the macro and microcirculation has been termed as a loss in hemodynamic coherence [3]. Hemodynamic incoherence is an independent factor of organ dysfunction and mortality. Additionally, microcirculation is the first circulatory system to be affected by shock and the last to improve [4].

Four types of microcirculatory alterations are described [3]:

a) Type 1: Flow heterogeneity is dominant in sepsis. It is characterized by stagnant capillaries circulating next to fast flowing ones. This causes a shunting of oxygen and a reduction in oxygen extraction capacity. b) Type 2: Decreased 02 capacity causes type 2 alterations. Excessive fluid resuscitation that causes hemodilution is a typical type 2 alteration.

c) Type 3: Microcirculatory stasis which is caused by the use of excess vasopressors or increased pressure in the venous end, such as fluid overload, leads to type 3 alterations.

d) Type 4: These are defined as a decrease in the density of the capillaries due to leakage. Tissue edema is a typical example of type 4 alteration (Figure 1).

Microcirculation assessment via hand-held vital microscopes (HVMs) provide insight into the pathophysiology of the shock. A clinician may interpret the findings as a deterioration in the convective and/or diffusive component of the oxygen transport to the tissue and manage the shock therapy accordingly. This explains why monitoring these two determinants of oxygen transport is essential in quantifying microcirculatory function [5,6]. In addition to monitoring oxygen transport, microcirculation assessment also gives details of inflammation and leukocyte activation [7]. Leukocytes can be tracked and counted. Thanks to the advancement in technology, the heavy workload imposed by previous generations of HVMs and software, is now past [8]. The newly introduced software can analyses 200 times faster compared to the gold standard. It is imminent that HVMs will be a close companion at the bedside in assessing and managing shock patients in the ICU. 


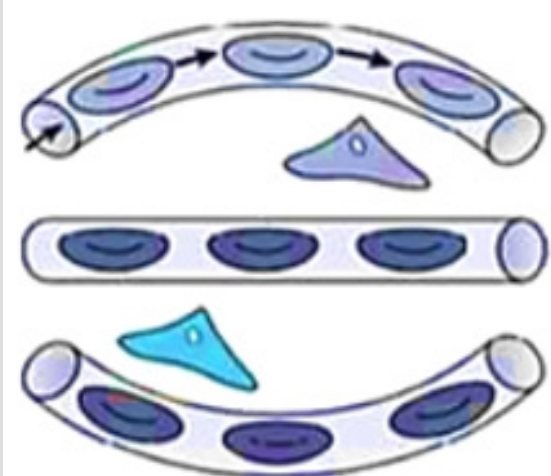

Type 1: Heterogeneity
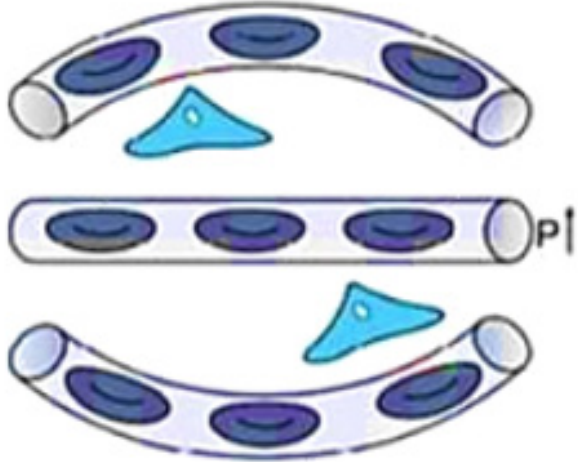

Type 3: Constriction/tamponade

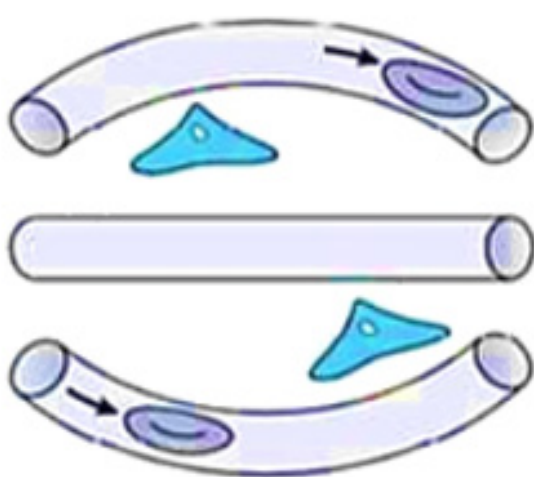

Type 2: Hemodilution
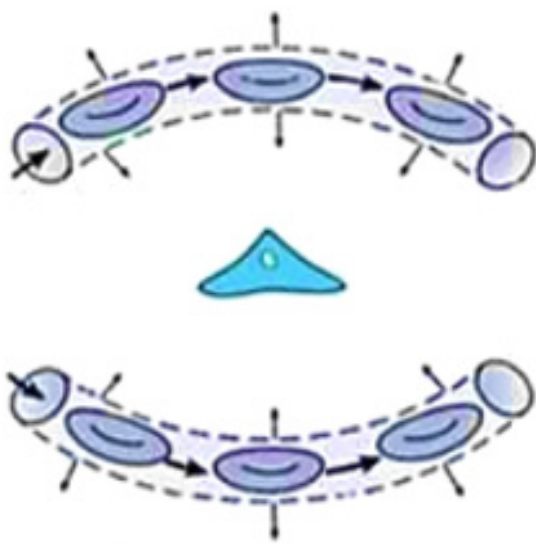

Type 4: Edema

Figure 1: Microcirculatory Alterations [3].

\section{References}

1. Gutterman DD, Chabowski DS, Kadlec AO, Durand MJ, Freed JK, et al (2016) The human microcirculation: regulation of flow and beyond Circulation research 118(1): 157-172.

2. Ince C, Boerma EC, Cecconi M, De Backer D, Shapiro NI, et al. (2018) Second consensus on the assessment of sublingual microcirculation in critically ill patients: results from a task force of the European Society of Intensive Care Medicine. Intensive care medicine 44(3): 281-299.

3. Ince C (2015) Hemodynamic coherence and the rationale for monitoring the microcirculation. Critical care 19(3): S8.

4. Sakr Y, Dubois MJ, De Backer D, Creteur J, Vincent JL (2004) Persistent microcirculatory alterations are associated with organ failure and death in patients with septic shock. Critical care medicine 32(9): 1825-1831.

ISSN: 2574-1241

DOI: 10.26717/BJSTR.2019.20.003439

Can Ince. Biomed J Sci \& Tech Res

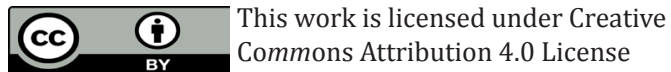

Submission Link: https://biomedres.us/submit-manuscript.php
5. Ince C (2014) The rationale for microcirculatory guided fluid therapy. Current opinion in critical care 20(3): 301-308.

6. Leach RM, Treacher DF (2002) The pulmonary physician in critical care 2: Oxygen delivery and consumption in the critically ill. Thorax 57(2): 170-177.

7. Uz Z, van Gulik TM, Aydemirli MD, Guerci P, Ince Y, et al. (2018) Identification and quantification of human microcirculatory leukocytes using handheld video microscopes at the bedside. Journal of Applied Physiology 124(6): 1550-1557.

8. Hilty MP, Guerci P, Ince Y, Toraman F, Ince C (2019) Microtools enables automated quantification of capillary density and red blood cell velocity in handheld vital microscopy. Communications Biology 2: 217.

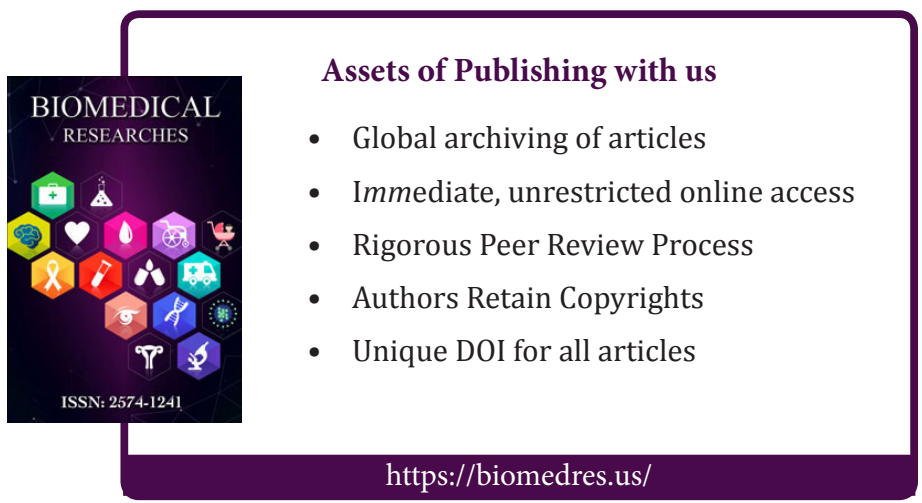

